\title{
Quedas em ambiente domiciliar: qualidade de vida dos idosos após as mesmas
}

\author{
Falls in the home environment: quality of life of the elderly after them \\ Caídas en el ambiente domiciliario: calidad de vida de los ancianos después de ellas
}

Recebido: 06/02/2022 | Revisado: 12/02/2022 | Aceito: 19/02/2022 | Publicado: 01/03/2022

\author{
Layane de Melo Ferreira \\ ORCID: https://orcid.org/0000-0002-2086-9235 \\ Faculdade Santa Maria, Brasil \\ E-mail: layane_melo_1988@icloud.com \\ Macerlane de Lira Silva \\ ORCID: https://orcid.org/0000-0002-9231-5477 \\ Faculdade Santa Maria, Brasil \\ E-mail: macerlane@ hotmail.com \\ Renata Livia Silva Fonsêca Moreira de Medeiros \\ ORCID: https://orcid.org/0000-0002-9913-4863 \\ Faculdade Santa Maria, Brasil \\ E-mail: renaliviamoreira@hotmail.com \\ Kelli Costa Souza \\ ORCID: https://orcid.org/0000-0002-1811-7499 \\ Faculdade Santa Maria, Brasil \\ E-mail: sulainecavalcante@outlook.com \\ Ankilma do Nasimento Andrade Feitosa \\ ORCID: https://orcid.org/0000-0002-4751-2404 \\ Faculdade Santa Maria, Brasil \\ E-mail: ankilmar@hotmail.com
}

\begin{abstract}
Resumo
Objetivos: Analisar as consequências das quedas na qualidade de vida dos idosos em ambiente domiciliar. Metodologia: A metodologia deste trabalho consiste, essencialmente, em pesquisa de ampla revisãobibliográfica. Os procedimentos de construção desses estudos pautarão em informações retiradas de artigos científicos publicados nos últimos 10 anos e pesquisados no Ministério da Saúde, nos bancos de dados Literatura Latino-americana e do Caribe Ciências da Saúde (LILACS), páginaeletrônica da Biblioteca Virtual em Saúde (BIREME) e Scientific Electronic Library Online (SciELO). Resultados e discussões: As quedas podem ter sérias consequências físicas e psicológicas, incluindo lesões, hospitalizações, perturbação da mobilidade, medo de cair novamente, restrição da atividade, declínio funcional, institucionalização e até mesmo a morte. Conclusão: As quedas em idosos são eventos multifatoriais, e o conhecimento de seus fatores de risco é importante para nortear o planejamento de medidas preventivas.
\end{abstract}

Palavras-chave: Saúde do idoso; Qualidade devida; Queda em idosos no ambiente domiciliar.

\begin{abstract}
Objectives: To analyze the consequences of falls on the quality of life of the elderly in the home environment. Methodology: The methodology of this work consists, essentially, of research with a broad bibliographic review. The construction procedures of these studies will be based on information taken from scientific articles published in the last 10 years and researched in the Ministry of Health, in the Latin American and Caribbean Literature databases Health Sciences (LILACS), electronic page of the Virtual Health Library (BIREME) and Scientific Electronic Library Online (SciELO). Results and discussion: Falls can have serious physical and psychological consequences, including injuries, hospitalizations, mobility disturbance, fear of falling again, activity restriction, functional decline, institutionalization and even death. Conclusion: Falls in the elderly are multifactorial events, and knowledge of their risk factors is important to guide the planning of preventive measures.
\end{abstract}

Keywords: Elderly health; Quality of life; Falls in the elderly in the home environment.

\section{Resumen}

Objetivos: analizar las consecuencias de las caídas en la calidad de vida de los ancianos en el ambiente domiciliario. Metodología: la metodología de este trabajo consiste, esencialmente, en una investigación con una amplia revisión bibliográfica. Los procedimientos de construcción de estos estudios se basarán en información extraída de artículos científicos publicados en los últimos 10 años e investigados en el ministerio de salud, en la base de datos de literatura latinoamericana y del caribe ciencias de la salud (LILACS), página electrónica de la biblioteca virtual en salud (Bireme) y biblioteca científica electrónica en línea (SCiELO). Resultados y discusión: las caídas pueden tener 
graves consecuencias físicas y psicológicas, incluyendo lesiones, hospitalizaciones, alteración de la movilidad, miedo a volver a caer, restricción de la actividad, deterioro funcional, institucionalización e incluso la muerte. Conclusión: las caídas en ancianos son eventos multifactoriales, y el conocimiento de sus factores de riesgo es importante para orientar la planificación de medidas preventivas.

Palabras clave: Salud del anciano; Calidad de vida; Caídas en el adulto mayor en el ámbito domiciliario.

\section{Introdução}

O envelhecimento é um processo importante, inerente a toda a humanidade, que traz consigo alguns aspectos que precisam ser levados em consideração para manutenção do bem-estar físico e social do idoso. Dessa forma, fica entendido que a velhice é uma etapa da vida singular, com aspectos próprios, e caracterizada como parte integrante de um ciclo natural, revelando-se na medida em que se efetiva como uma experiência única e diferenciada a cada sujeito (Marchi et al., 2016).

Avaliando a trajetória de vida natural de cada indivíduo, fica evidente a percepção de que, durante o percurso existencial, o trabalho é que move as pessoas, proporcionando vitalidade, interação e dinamicidade, no que concerne ao estabelecimento de metas a serem alcançadas, dificuldades a serem transpostas e objetivos a serem seguidos. Ao atingir a idade requerida pelo Ministério do Trabalho, associados aos Sindicatos e Associações de Trabalhadores em suas diversas categorias, e também órgãos como Instituto Nacionais de Seguros Social (INSS), homens com 65 anos, mulheres com 60 anos conseguem a tão sonhadaaposentadoria, sendo vista como repouso, descanso remunerado pelos longos anos de labuta (Araldi et al., 2017).

Para Thumé et al. (2018), devido ao aumento da expectativa de vida no Brasil, a frequência do uso de drogas por idosos é muito alta, e aumenta a cada dia. Alguns desses medicamentos podem causar efeitos colaterais quando usados, como tontura e reflexos enfraquecidos, que podem causar quedas e subsequentes fraturas.

O idoso deve gerenciar suas atividades sob a premissa de manter um equilíbrio entre potencialidades e limitações, o que é inevitável no processo de envelhecimento. Diante dessa premissa, é muito comum o idoso começar a ser acometido por doenças crônicas a partir de uma determinada idade, mas consegue manter sua qualidade de vida por meio da criatividade edo lazer, sem abrir mão dos cuidados médicos (Borges et al., 2010).

De acordo com Oliveira (2019), o evento relacionado a quedas em idosos é muito comum, resultado de diversas causas, mas que estão atrelados a graves consequências físicas, psicológicas e sociais, que determinam as complicações que impactam negativamente na qualidade de vida dessas pessoas. Pode ser evitado, tomando medidas preventivas adequadas, identificando a causa e formulando formas de reduzir sua ocorrência.

As pesquisas empreendidas por Muniz et al. (2019), revelam uma iminente preocupação com o aumento da expectativa de vida das pessoas acima de 70 anos que não possuem uma qualidade de vida considerada regular, pois acontecimentos como tombos e quedas dentro de casa comprometeram o bem-estar dos mesmos, resultando em transtornos de ordem física, psicológica e social.

Para que seja assegurada a autonomia do idoso e resguardada a sua integridade funcional, faz-se necessário um planejamento sobre o ambiente em que ele vive, lançando estratégias paraque não haja armadilhas ou obstáculos que venham a provocar acidentes domésticos. Nesse sentido, a casa deve ser livre de qualquer espaço amontoado, escorregadio, sem objetos espalhados pelo chão. Somente assim, o bem-estar de cada pessoa na terceira idade será conservado.

Dessa forma, a rotina de vida do idoso acaba recebendo influências negativas provenientes dos resultados de quedas, pois limita seus movimentos e, consequentemente, torna complexa sua circulação e movimentação no contexto domiciliar. O resultado de tombos ou acidentes que ocorrem dentro de sua própria residência compromete atividades 
simples como deitar e levantar-se, caminhar em superfície plana, realizar higiene pessoal e ter uma vida socialativa (Borges $e t$ al., 2010).

Segundo os estudos de Silva et al. (2017), as quedas são um importante fator causal dos idosos,pois estão associadas a um alto nível de deficiência e mortalidade. O processo de envelhecimento causa muitas mudanças no corpo humano, que pode fazer com que o indivíduo experimente, em sua vida diária, ao longo do tempo, a necessidade de ajuda pararealizar a vida. As quedas, nesta faixa etária, podem ser consideradas importantes fatores de alerta, a fim de evitar as sequelas físicas e emocionais decorrentes desses eventos.

O assunto estudado é uma fonte de preocupação, especialmente quando as pessoas descrevem este evento como normal e característico do envelhecimento. Foi verificado, em serviços de emergência, que as quedas são uma ocorrência comum e causamlesões, a principal etiologia das mortes acidentais em pessoas com mais de 65 anos de idade (Martins $e t$ al., 2019).

O crescimento da população idosa chama a atenção dos profissionais de saúde para o bem-estar desse grupo sensível a quedas.

A construção do texto será valiosa para a formação profissional dos que irão atuar no campo da enfermagem, enquanto área que possibilita a melhoria na qualidade de vida dos idosos, a partir do seu ingresso na atenção primária de saúde.

A justificativa para a escolha deste tema se deve à relevância social, visto que o envelhecimento da população mundial é um fato irreversível, que se tornará mais acentuado, daí a importância do planejamento promoção da saúde específica para essa faixa etária.

Diante do esposto, o objetivo é analisar as consequências das quedas na qualidade de vida dos idosos em ambiente domiciliar.

\section{Metodologia}

A metodologia deste trabalho consiste, essencialmente, em pesquisa de ampla revisão bibliográfica. A presente pesquisa bibliográfica fará a análise do tema, desenvolvendo uma aferição crítica e catalogando materiais através de artigos científicos, livros, dentre outros recursos que tratem do assunto. (Souza et al., 2010).

Nesse sentido, a pergunta norteadora desse estudo é saber como e em que medida as quedas em idosos no ambiente domiciliar afetam sua qualidade de vida?

Os procedimentos de construção desses estudos pautarão em informações retiradas de artigos científicos publicados nos últimos 10 anos e pesquisados no Ministério da Saúde, nos bancos de dados Literatura Latino-americana e do Caribe Ciências da Saúde (LILACS), páginaeletrônica da Biblioteca Virtual em Saúde (BIREME) e Scientific Electronic Library Online (SciELO).

Para a construção deste trabalho, algumas etapas serão fundamentais, como a escolha do tema e da questão norteadora. Posteriormente, foram estabelecidos os critérios de inclusão e exclusão. Em seguida, foi feita a extração das informações e resultados relevantes. Na etapa posterior, ocorreu a leitura e análise de forma crítica e sistemática dos estudos. Nas etapas finais, foram feitas a interpretação e discussão dos resultados e, posteriormente, apresentada uma síntese do conhecimento adquirido.

Como critérios de inclusão foram catalogados estudos de 2010 a 2021, de artigos quedelimitassem os objetivos do referido trabalho. Serão incluídos estudos de língua portuguesa, principalmente, além de inglês. Os critérios de exclusão serão artigos não condizentes com a objetividade do estudo, além de publicações anteriores a 2015. 
Os descritores utilizados para a busca dos artigos foram: saúde do idoso, qualidade de vida e queda em idosos no ambiente domiciliar.

Para realizar a efetiva análise dos artigos e obras serão utilizados quadros e tabelas, nas quais abordarão os aspectos: Título da pesquisa/autores, base de dados, ano de publicação/periódico, modalidade da pesquisa, objetivo e principais resultados.

Posteriormente, ao estudo e leitura dos artigos selecionados, intenciona-se, através da análise dos resultados, responder a questão que é eixo condutor desse trabalho.

Diante desse estudo, no que se refere às bases citadas, serão selecionados trabalhos quese enquadrem com a questão norteadora e características aqui citadas anteriormente, que serão discutidos e demonstrados minuciosamente.

\section{Resultados}

No quadro a seguir apresenta o panorama geral dos artigos escolhidos para compor a amostra da revisão, sendo sete artigos, um do ano de 2019; dois do ano de 2017; um do ano de 2015; um do ano de 2013; e um do ano de 2010 (alguns artigos encontravam-se repetidos dentro das bases de dados utilizadas).

Quadro 1: Artigos analisados e incluídos na revisão.

\begin{tabular}{|c|c|c|c|c|c|}
\hline $\begin{array}{c}\text { Base de } \\
\text { Dados }\end{array}$ & Título & Autores & $\begin{array}{c}\text { Ano de } \\
\text { Publicação }\end{array}$ & Objetivos & Achados \\
\hline SCIELO & $\begin{array}{l}\text { Urgência geriátrica: a } \\
\text { influência da adaptação } \\
\text { do ambiente domiciliar } \\
\text { na prevenção de } \\
\text { quedas. }\end{array}$ & $\begin{array}{l}\text { RIBEIRO, D.R, } \\
\text { et al. }\end{array}$ & 2019 & $\begin{array}{lr}\text { Descrever } & \text { a } \\
\text { influência } & \text { da } \\
\text { adaptação } & \text { do } \\
\text { ambiente } & \\
\text { domiciliar } & \text { na } \\
\text { prevenção } & \text { de } \\
\text { quedas } & \text { em } \\
\text { idosos. } & \end{array}$ & $\begin{array}{l}\text { A queda nos idosos é um evento } \\
\text { multifatorial em que existe uma combinação } \\
\text { de fatores intrínsecos e extrínsecos. As } \\
\text { principais causas estão relacionadas a } \\
\text { fatores ambientais e fisiológicos, tendo } \\
\text { como exemplo: ausência de iluminação nos } \\
\text { ambientes; pisos escorregadios; escadas sem } \\
\text { corrimão; vasos sanitários; cadeiras e cama } \\
\text { muito baixos e sem apoio para sentar e } \\
\text { levantar; ausência de barras de apoio no } \\
\text { banheiro; obstáculos no caminho, como } \\
\text { fios; presença de animais domésticos; } \\
\text { móveis baixos; dispositivo auxiliar para } \\
\text { marcha danificada; doenças que afetam a } \\
\text { visão; perda gradativa da força muscular e } \\
\text { elasticidade; diminuição do sistema } \\
\text { sensorial e nervoso; alteração da } \\
\text { estabilidade e dinâmica articular; } \\
\text { comprometimento da postura, marcha e } \\
\text { equilíbrio. }\end{array}$ \\
\hline BIREME & $\begin{array}{l}\text { Quedas em idosos e } \\
\text { comorbidades clínicas. }\end{array}$ & $\begin{array}{l}\text { LIMA, D.A., et } \\
\text { al. }\end{array}$ & 2013 & $\begin{array}{lr}\text { Analisar } & \text { os } \\
\text { fatores de } & \text { risco } \\
\text { associados } & \text { a } \\
\text { quedas } & \text { em } \\
\text { idosos. } & \end{array}$ & $\begin{array}{l}\text { Os fatores encontrados na literatura, mais } \\
\text { comumente associados às quedas em idosos, } \\
\text { foram: idade avançada, sedentarismo, maior } \\
\text { número de medicações de uso contínuo, uso } \\
\text { de benzodiazepínicos, presença de doenças } \\
\text { crônicas - inclusive as demenciais -, } \\
\text { comprometimento da acuidade visual, } \\
\text { antecedentes de quedas, autopercepção de } \\
\text { saúde ruim, depressão, obstáculos no } \\
\text { domicílio, presença de alterações de marcha }\end{array}$ \\
\hline
\end{tabular}




\begin{tabular}{|c|c|c|c|c|c|}
\hline & & & & & e de equilíbrio. \\
\hline & $\begin{array}{l}\text { Causas e consequências } \\
\text { de quedas de idosos em } \\
\text { domicílio. }\end{array}$ & & & $\begin{array}{l}\text { Causas e } \\
\text { consequências de } \\
\text { quedas em idosos } \\
\text { em domicílio, } \\
\text { descrevendo o } \\
\text { ambiente mais } \\
\text { propício a } \\
\text { quedas, com suas } \\
\text { consequências, o } \\
\text { que } \\
\text { impossibilitam as } \\
\text { capacidades } \\
\text { funcionais. }\end{array}$ & $\begin{array}{l}\text { São considerados determinantes na } \\
\text { ocorrência de quedas na população idosa } \\
\text { fatores intrínsecos, através de alterações nos } \\
\text { sistemas musculoesqueléticas, vestibular, } \\
\text { sensorial e cognitivo, e os fatores } \\
\text { extrínsecos relacionados ao ambiente. É } \\
\text { importante considerar alterações no } \\
\text { domicílio que o tornem um ambiente mais } \\
\text { saudável e seguro, facilitando a } \\
\text { deambulação dos idosos e o } \\
\text { desenvolvimento de atividades de vida } \\
\text { diárias, diminuindo o risco de quedas. }\end{array}$ \\
\hline LILACS & $\begin{array}{l}\text { Análise de similitude } \\
\text { dos fatores associados } \\
\text { à queda de idosos. }\end{array}$ & $\begin{array}{l}\text { MOURA, } \\
\text { S.R.B., et al. }\end{array}$ & 2015 & $\begin{array}{l}\text { Fazer uma análise } \\
\text { de similitude dos } \\
\text { fatores } \\
\text { associados à } \\
\text { queda de idosos. }\end{array}$ & $\begin{array}{l}\text { As quedas entre idosos é um problema que } \\
\text { pode ser irreversível caso ocorra fraturas } \\
\text { graves. Podem afetar a qualidade de vida } \\
\text { dos idosos por causar imobilidade e } \\
\text { dependência. Há necessidade de melhorar a } \\
\text { política pública de atenção ao idoso, } \\
\text { diminuindo os fatores de risco associados às } \\
\text { quedas. }\end{array}$ \\
\hline LILACS & $\begin{array}{l}\text { Fatores relacionados à } \\
\text { ocorrência de queda de } \\
\text { idosos. }\end{array}$ & $\begin{array}{l}\text { BEIJOS, L.A., et } \\
\text { al. }\end{array}$ & 2017 & $\begin{array}{l}\text { Verificar se } \\
\text { fatores } \\
\text { fisiológicos, } \\
\text { ambientais } \\
\text { cognitivos que } \\
\text { influenciam na } \\
\text { ocorrência de } \\
\text { quedas de idosos. }\end{array}$ & $\begin{array}{l}\text { Percebe-se que as quedas fazem com } \\
\text { que os idosos percam sua autonomia e a } \\
\text { qualidade de vida, e isso repercute em seus } \\
\text { cuidadores, principalmente em seus } \\
\text { familiares, mudando suas rotinas para } \\
\text { adaptar-se aos cuidados especiais que os } \\
\text { idosos necessitam após a queda. Quedas } \\
\text { podem provocar fraturas, sendo que, após a } \\
\text { fratura, o risco de óbito aumenta } \\
\text { significativamente. }\end{array}$ \\
\hline SCIELO & $\begin{array}{l}\text { O olhar e o sentir do } \\
\text { idoso no pós-queda. }\end{array}$ & $\begin{array}{l}\text { CARVALHO, } \\
\text { E.M.R., et al. }\end{array}$ & 2010 & $\begin{array}{l}\text { Propôs a uma } \\
\text { melhor } \\
\text { compreensão do } \\
\text { que se passa com } \\
\text { esta população } \\
\text { após a queda, } \\
\text { além de estreitar } \\
\text { as relações entre } \\
\text { a equipe de saúde } \\
\text { e o idoso } \\
\text { fragilizado, pela } \\
\text { possível } \\
\text { dependência que } \\
\text { a queda lhe } \\
\text { impõe. }\end{array}$ & $\begin{array}{l}\text { Percebe-se que, entre os idosos } \\
\text { investigados, existe um processo de } \\
\text { passividade em aceitar o envelhecer, e que a } \\
\text { queda poderá causar diminuição da } \\
\text { capacidade funcional e da autoestima. } \\
\text { Tornam-se necessários, portanto, programas } \\
\text { de prevenção a quedas e incentivo ao } \\
\text { envelhecimento ativo. }\end{array}$ \\
\hline BIREME & $\begin{array}{lr}\text { Qualidade de } & \text { vida em } \\
\text { idosos que } & \text { sofreram } \\
\text { quedas: } & \text { revisão }\end{array}$ & $\begin{array}{l}\text { NICOLUSSI, A. } \\
\text { C., et al. }\end{array}$ & 2012 & $\begin{array}{l}\text { Avaliar } \\
\text { conhecimento } \\
\text { científico } \\
\text { produzido, }\end{array}$ & $\begin{array}{l}\text { Encontrou-se que as quedas são frequentes } \\
\text { nos idosos, sendo que aqueles com mais } \\
\text { fatores de risco intrínsecos, e que já caíram, } \\
\text { apresentaram mais medo e possuem mais } \\
\text { chance de caírem novamente; e também } \\
\text { relataram déficits nas funções física, }\end{array}$ \\
\hline
\end{tabular}




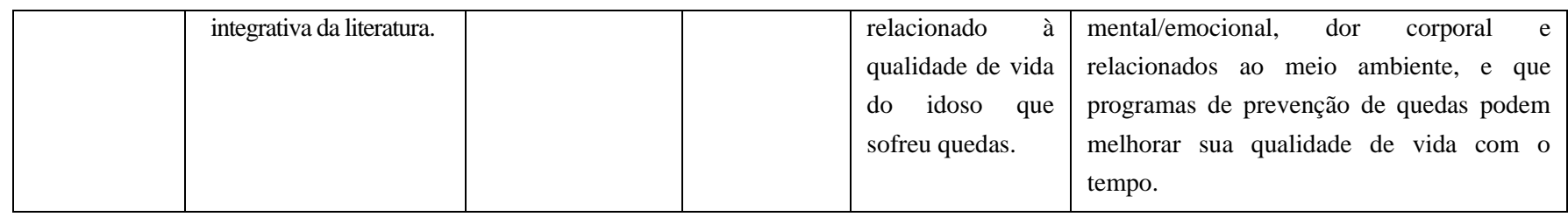

Fonte: Autores (2022).

\section{Discussões}

A queda nos idosos é um evento multifatorial em que existe uma combinação de fatores intrínsecos e extrínsecos. As principais causas estão relacionadas a fatores ambientais e fisiológicos, tendo como exemplo: ausência de iluminação nos ambientes; pisos escorregadios; escadas sem corrimão; vasos sanitários; cadeiras e cama muito baixas e sem apoio para sentar e levantar; ausência de barras de apoio no banheiro; obstáculos no caminho, como fios; presença de animais domésticos; móveis baixos; dispositivo auxiliar para marcha danificada; doenças que afetam a visão; perda gradativa da força muscular e elasticidade; diminuição do sistema sensorial e nervoso; alteração da estabilidade e dinâmica articular; comprometimento da postura, marcha e equilíbrio. (Feitosa et al., 2020).

As quedas acometem, frequentemente, mais mulheres por maior prevalência de doenças crônicas, maior fragilidade destas em relação aos homens, assim como se suspeita, ainda, que o fato pode estar relacionado a uma maior exposição a atividades domésticas e a um comportamento de maior risco. (Lopes et al., 2007). Como também mulheres que são diagnosticadas com osteoporose e idade avançada estão mais vulneráveis à queda e tem como maior consequência fraturas no fêmur e pé. Os usos de alguns medicamentos também podem favorecer a ocorrência de quedas, por conta de alguns efeitos colaterais dos medicamentos, como os benzodiazepínicos, que causam efeitos sedativos, e os diuréticos, que causam hipocalcemia e hipotensão, podendo ocorrer tonturas, aumentando o risco para queda. (Jahana et al., 2007).

As quedas mais graves geram fraturas tanto em membro superior como inferior, podendo deixar o idoso acamado e incapaz de realizar suas atividades diárias, como também podem levar à morte. (Freitas et al., 2010). As consequências vão além de agravos físicos, como ferimentos e fraturas, ocorrem também consequências psicoemocionais como o medo de cair novamente, que interferem na sua mobilidade, no qual alguns idosos se privam de determinadas atividades, como ir ao banheiro, de frequentar certos ambientes do domicilio, o que torna o idoso cada vez mais dependente. (Medeiros et al., 2017).

No idoso, os sistemas responsáveis pela estabilidade postural estão afetados, e diversas etapas do controle postural podem se encontrar suprimidas, diminuindo a capacidade de resposta e de compensação, levando a um aumento da instabilidade. (Brasil et al., 2010).

Algumas doenças promovem alterações na capacidade do andar dos idosos. Estas alterações se apresentam de acordo com quatro categorias funcionais. São elas: deformidades, fraqueza muscular, falta de controle e dor. Cada categoria possui uma característica própria, que pode levar a um prejuízo funcional. O reconhecimento dessas alterações permite uma avaliação criteriosa e diferenciada entre o que é déficit primário e o que é resultado de ações compensatórias. (Araújo et al., 2017).

Os fatores de risco de quedas em idosos nos domicílios encontrados e/ou analisados nos estudos selecionados foram: alterações cognitivas, acuidade visual, ambiente domiciliar (luz, disposição de móveis e objetos, pisos, ausência de barra de apoio), diminuição da força física de membros superiores e inferiores, autopercepção da condição de saúde e osteoporose, sendo identificados maiores riscos de quedas entre os idosos de idade mais avançada, mulheres e entre aqueles com maior dependência de cuidados. (Aveiro et al., 2011).

Ao que se refere ao momento das quedas, identificou-se que elas ocorrem principalmente no quintal, no banheiro, e ao caminhar acontece o tropeço. Para um outro autor, os perigos no ambiente serão maiores quanto mais elevado for o grau de 
vulnerabilidade do idoso e a consequência que este problema poderá causar. Justifica ainda que os idosos não caem por realizações de atividades perigosas (subir em cadeiras ou escadas), e sim em realizações de atividades rotineiras. (Duarte $e t$ al., 2016).

Os estudos apontam que os programas de gestão integrada de riscos que enfatizam várias intervenções, incluindo a avaliação por profissional capacitado em gerontologia e geriatria, com as devidas modificações nos domicílios, são mais eficazes para reduzir as quedas entre os idosos nas residências. As condições adequadas são importantes para minimizar os fatores de risco, levando em considerações as adequações para todos os ambientes da casa e dos acessórios que devem e não devem constar nos respectivos cômodos, podendo evitar fraturas e internações. (Borges et al., 2010).

As quedas podem ter sérias consequências físicas e psicológicas, incluindo lesões, hospitalizações, perturbação da mobilidade, medo de cair novamente, restrição da atividade, declínio funcional, institucionalização e até mesmo a morte. (Medeiro et al., 2017).

O envelhecimento é um processo natural que todo ser humano sofre. Acontece que, nesta fase, a força muscular, a flexibilidade, o equilíbrio e as articulações começam a apresentar uma perda considerável de tônus muscular, podendo ocasionar quedas e problemas de saúde significativos para o idoso. Dessa forma, atividade de prevenção, como os exercícios de fortalecimento muscular, treino proprioceptivos e de equilíbrio favorecem as capacidades funcionais, nas quais estão inseridas a resistência e flexibilidades necessárias à boa qualidade de vida da saúde do idoso. (Marchi et al., 2016).

A perda de autonomia e independência traz a necessidade de auxílio dos familiares e/ou cuidadores, bem como uma equipe interdisciplinar para o desenvolvimento de atividades cotidianas, assim como, é importante enfatizar, que a família precisa ser orientada quanto ao processo de envelhecimento e também sobre os problemas relacionados a ele. (Feitosa et al., 2020).

Esses dados justificam que as quedas em domicilio são constantes e muito comuns. Diante deste cenário, o sistema de saúde e seus profissionais precisam estar preparados para atuarem na prevenção de quedas e no tratamento e reabilitação de suas consequências. (Araújo et al., 2017).

\section{Conclusão}

As quedas em idosos são eventos multifatoriais, e o conhecimento de seus fatores de risco é importante para nortear o planejamento de medidas preventivas. Os objetivos de tais medidas, tanto do ponto de vista de intervenções individuais quanto do de políticas públicas são evitar as quedas ou reduzir seu número, além de preservar a funcionalidade e melhorar a qualidade de vida.

\section{Referências}

Araújo, L.A. (2017). O Programa Saúde da Família: perfil de idosos assistidos por uma equipe. Rev. Bras. Enferm., Brasília , v. 57, n. 5, out.

Araldi, M. (2017). A descoberta de projetos de vida: contribuição do projeto idoso empreendedor no processo de envelhecimento. Trabalho de Conclusão de Curso de Serviço Social, UFSC. Florianópolis.

Aveiro, M. S. et al. (2011). Perspectivas da participação do fisioterapeuta no Programa Saúde da Família na atenção à saúde do idoso. Ciência \& Saúde Coletiva, v.16, supl.1, p.1467-78.

Borges, A. M. P. et al. (2010). A contribuição do fisioterapeuta para o Programa de Saúde da Família - uma revisão da literatura. UNICiências, Cuiabá, v. 14, n. 1, p. $69-82$.

Brasil,(2005). Conselho Federal de Fisioterapia e Terapia Ocupacional. Saúde da Família: uma nova opção para o trabalho do fisioterapeuta e terapeuta ocupacional. Revista trimestral do COFFITO, Brasília, v. 7, n. 24, p. 6-8.

Brasil. (2019). Lei no 10.741de 01 de outubro de 2003. Estatuto do idoso. www.planalto.org . 
Brasil. (2019). Ministério da Saúde. Portaria nº 2.528 de 19 de outubro de 2006. Aprova a PolíticaNacional de Saúde da Pessoa Idosa. Diário Oficial da União, Brasília, 19 de outubro de 2006. www.planalto.org.

Brasil. (1998). Ministério da Saúde. Saúde da família: uma estratégia para a reorientação do modeloassistencial. Brasília: Ministério da Saúde.

Brasil. (2006). Ministério da Saúde. Portaria GM n. 648, de 28 de Março de 2006. Aprova a Política Nacional de Atenção Básica, estabelecendo a revisão de diretrizes e normas para a organização da Atenção Básica para o Programa Saúde da Família (PSF) e o Programa AgentesComunitários de Saúde (PACS). Brasília: Ministério da Saúde.

Brasil. (2010). Ministério da Saúde. Portaria GM/MS n. 4.279, de 30 de Dezembro de 2010. Estabelece diretrizes para a organização da Rede de Atenção à Saúde no âmbito do Sistema Único de Saúde (SUS). Brasília: Ministério da Saúde.

Brasil. (2006). Ministério da Saúde. Regionalização solidária e cooperativa: orientação para sua implementação no SUS. Brasília: Ministério da Saúde, b. 40 p. (Série Pactos Pela Saúde, v. 3).

Brasil. (2011). Ministério da Saúde. Secretaria Executiva. Departamento de Monitoramento e Avaliação o SUS. Programa de Avaliação para Qualificação do Sistema Único de Saúde. Brasília, DF.

Conill, E. M. (2008). Ensaio histórico-conceitual sobre a Atenção Primária à Saúde: desafios para a organização de serviços básicos e da estratégia saúde da família em centros urbanos no Brasil. Cadernos de Saúde Pública, Rio de Janeiro, v. 24, supl. 1, p. 7-16.

Duarte, Y. A. (2016). Atendimento Domiciliar: Um Enfoque Gerontológico. 1 ed. São Paulo: Ateneu.

Feitosa, A. N. A. et al. (2020). O Processo de Trabalho do Enfermeiro na Atenção Básica: Gerenciamento e Assistência: The Nurse's Work Process In Basic Care: Management And Assistance. Brazilian Journal of Production Engineering-BJPE, p. 199- 207.

Lakatos, E. M. \& Marconi, M. A. (2006). Metodologia do Trabalho Científico: procedimentos básicos, pesquisa bibliográfica, projeto e relatório publicações e trabalhos científicos. $6^{\circ}$ ed. $7^{\circ}$ reimpr. São Paulo: Atlas.

Magalhães, L. C. et al. (2003). Estudo comparativo sobre o desempenho perceptual e motor na idade escolar em crianças nascidas pré-termo e a termo. Revista Arquivos de NeuroPsiquiatria, São Paulo, v. 61, n. 2A, p. 250-255.

Marchi, R. (2016). Escolhendo Qualidade de Vida: Opção Saúde. CPH - Tecnologia emSaúde.

Medeiros. (2017). Aline Carolina. Qualidade de Vida: Um desafio para os aposentados da CELESC. Trabalho de Conclusão de Curso - Serviço Social UFSC.

Milnitzky, C. \& Sung, F. \& Pereira, R.M. (2017). Políticas públicas e envelhecimento: conquistas e desafios, envelhecimento e a bioética: o respeito a autonomia doidoso. A Terceira Idade, v. 15, n. 31, São Paulo.

Minayo, M. C. S. \& Sanches, O. (1993). Quantitativo-qualitativo: oposição ou Complementaridade? Cad. Saúde Pública, São Paulo, v. 9, n. 3, p. 239-262.

Martins, K. M. S. \& Walquiria L. \& Álvares, A. C. M. A. (2019). Importância da imunização: revisão integrativa. Revista de Iniciação Científica e Extensão, v. 2, n. 2, p. 28-33.

Mendes, E. V. Agora mais do que nunca - uma revisão bibliográfica sobre Atenção Primáriaà Saúde. In: conselho nacional de secretários de saúde. Oficinas de Planificação da Atenção Primária à Saúde nos Estados. Brasília: CONASS, 2009.

Muniz, C. F.et al. (2007). Projeto de assistência interdisciplinar ao idoso em nível primário: Enfoque dos alunos de fisioterapia. APS. v.10, ${ }^{\circ}$ 1, p. 84-89, jan./jun. 2007 http:// www.ufj.br/nates/files/2009/12/Pidoso.pdf>

Neri, A. L. (2015). Qualidade de Vida e Idade Madura. Campinas: Papirus.

Oliveira, (2015). Reinaldo Marcon. Trabalhar com famílias no Programa de Saúde da Família: a prática do enfermeiro em Maringá- Paraná. Rev Esc Enferm USP.

Oms - organização mundial de saúde. (1978). Declaração de Alma-Alta. Alma-Alta: OMS, 1978. 3 p. <http://www.opas.org.br/coletiva/uploadArq/AlmaAta.pdf $>$.

Oms - organização mundial de saúde. (2008). Relatório Mundial da Saúde 2008: Cuidados de Saúde Primários - Agora mais que nunca. Genebra: OMS.

Organização pan-americana da saúde - opas. (2011). A atenção à saúde coordenadapela APS: construindo as redes de atenção no SUS - Contribuições para o debate. Brasília: OPAS.

Oliveira, Valéria Conceição et al. (2019). A percepção da equipe de enfermagem sobre a segurançado paciente em sala de vacinação. Revista Cuidarte, v. 10, n. 1 .

Silva,M. J. (2017). O autocuidado dos idosos: intervenção de enfermagem e melhor qualidadede vida. Rev Enfem UERJ.

Simões, R. (2017). Qual idade de vida na qual idade de vida. In: MOREIRA, W. W.(org.) Qualidade de vida: complexidade e educação. Campinas: Papirus.

Thumé, E. C. et al. (2016). Assistência Domiciliar a Idosos: Fatores Associados, Características do Acesso e do Cuidado. Rev. Saúde Pública, v.44, n.6, 2016.:http://www.scielosp.org/pdf/rsp/v44n6/1961.pdf 